\title{
Potential Value of miR-23a for Discriminating Neuromyelitis Optica Spectrum Disorder from Multiple Sclerosis
} \author{
Mona Essawi, MD ${ }^{1}$ \\ 'Medical Molecular Genetics Department, National Research Centre, Cairo, Egypt \\ ${ }^{2}$ Neurology Department, Faculty of Medicine, Cairo University, Cairo, Egypt \\ ${ }^{3}$ Rheumatology Department, Faculty of Medicine, Cairo University, Cairo, Egypt \\ ${ }^{4}$ Department of Community Medicine, National Research Centre, Cairo, Egypt
}

Wessam Sharaf-Eldin, $\mathrm{PhD}^{1 *}$; Nirmeen Kishk, MD²; Basma Sakr, MSc ${ }^{3}$; Hazem El-Hariri, MSc ${ }^{4}$; Miral Refeat, PhD ${ }^{1}$; Nagham ElBagoury, PhD ${ }^{1}$;

\begin{abstract}
Background: Until now, no laboratory test or test set can guarantee the diagnosis of multiple sclerosis (MS) at early disease stages, and the disease symptoms may interfere with many other disease conditions. Analyzing the expression of circulating miRNAs may provide a useful approach for early and differential MS diagnosis. The main objective is assessment of the potential of serum miR23a, miR-155, and miR-572 to differentiate between MS and other neuroinflammatory diseases.

Methods: Serum miRNAs were obtained from 37 MS patients and 25 healthy age-matched controls, along with patients with neuromyelitis optica spectrum disorder (NMOSD) $[n=13$ ] and neuropsychiatric systemic lupus erythematosus (NPSLE) [ $n=10]$. miRNA expression levels were analyzed using real-time polymerase chain reaction (PCR) and pairwise comparisons were made to reveal the diagnostic/distinguishing potential of the analyzed miRNAs.

Results: In the study cohort, the three investigated miRNAs failed to display significant dysregulation in MS patients. However, they could significantly discriminate patients with NMOSD and NPSLE [median (IQR): 8.1 (6.1-9.2) and 8.8 (7.9-9.7) for miR-23a, 7.5 (5.3-8.3) and 8.0 (7.5-9.5) for miR-155 and 6.9 (5.0-8.8) and 6.4 (5.3-8.8) for miR-572 in NMOSD and NPSLE, respectively] from healthy subjects [median (IQR): 3.4 (1.5-4.3), 3.1 (1.1-5.6) and 3.5 (1.7-5.6) for miR-23a, miR-155 and miR-572, respectively], with area under the curve $(A \cup C) \leq 0.8$. Remarkably, miR-23a has been emerging as a prospective biomarker for differentiation of MS from NMOSD as well as NPSLE (AUC<0.9). The miRNA combined use contributed to enhanced diagnostic and discriminatory performance in the study groups.

Conclusion: Certain miRNA expression levels would contribute to discriminating MS from other neuroinflammatory diseases. Keywords: Biomarkers, MicroRNAs, Multiple sclerosis, Neuroinflammatory

Cite this article as: Sharaf-Eldin W, Kishk N, Sakr B, El-Hariri H, Refeat M, ElBagoury N, et al. Potential value of miR-23a for discriminating neuromyelitis optica spectrum disorder from multiple sclerosis. Arch Iran Med. 2020;23(10):678-687. doi: 10.34172/aim.2020.86
\end{abstract}

Received: October 6, 2019, Accepted: March 14, 2020, ePublished: October 1, 2020

\section{Introduction}

Multiple sclerosis (MS) represents the most common neurologic disease in young adults, severely reducing the patient's quality of life and their work productivity and imposing tremendous economic burdens on the individual, family and society. About $80 \%$ of MS patients present with a relapsing-remitting (RR) disease form. This clinical category will be subsequently followed by secondary progressive MS (SPMS) in the majority of patients. Other disease forms include primary progressive (PP), and to a lesser extent, progressive relapsing MS (PRMS). ${ }^{1}$

A general increase in MS incidence has been reported in the last decades. ${ }^{2}$ The calculated global median prevalence seemed to increase from 30 (in 2008) to 33 per 100000 (in 2013). ${ }^{3}$ The Middle East and North Africa are considered a moderate to high risk region for the disease. ${ }^{4}$ In Egypt, MS represents $1.4 \%$ out of all neurological disorders and its prevalence was recently estimated to be 13.7 per 100000 individuals. 5

Early diagnosis of MS is critical for optimal patient management, counseling and therapy. Therefore, it is imperative to identify accurate and reliable diagnostic biomarkers for MS during the early disease course ${ }^{6}$. However, the cryptic nature of the disease during early stages, the extensive phenotypic variability and the clinical overlap with several other diseases make MS diagnosis more challenging. Importantly, it has been reported that MS diagnosis was delayed up to 2 years in about $28 \%$ of Egyptian patients.

miRNA dysregulation has been associated with many diseases, including MS. Several studies have proposed that the dysregulated miRNAs in MS may serve as diagnostic biomarkers and/or targets for new therapeutic interventions. In particular, the expression levels of 
miR-23a, miR-155, and miR-572 were shown to be dysregulated in several compartments of MS patients. miR-23a levels were shown to be decreased in the serum ${ }^{8,9}$ as well as the total subset of T-cells of MS patients. ${ }^{10}$ However, it was significantly upregulated in peripheral blood mononuclear cells (PBMCs) of patients with RRMS and PPMS $^{9}$ and active and inactive brain lesions from MS patients. ${ }^{11}$ On the other hand, miR-155 was upregulated in sera of patients with MS, ${ }^{12}$ PBMCs of RRMS patients, ${ }^{13}$ peripheral circulating CD14+ monocytes of MS patients as well as CD68+ microglial cells in active MS brain lesions, ${ }^{14}$ the neurovascular unit of MS brains and EAE spinal cords, ${ }^{15}$ active brain lesions from MS patients ${ }^{11}$ and MS cerebral white matter. ${ }^{16}$ Regarding miR-572, it is reportedly upregulated in plasma of MS individuals ${ }^{17}$ and serum of SPMS and RRMS during relapse, while it is downregulated in PPMS and in the remission phase of RRMS. ${ }^{18}$ The current study was conducted to evaluate the potential value of these miRNAs as molecular biomarkers for differential diagnosis of MS.

\section{Materials and Methods}

Subjects

This study included 37 patients with MS (18 RR in the remitting phase and 19 SP), 13 with neuromyelitis optica spectrum disorder (NMOSD) and 10 with NPSLE. The diagnoses of MS, NMOSD and NPSLE were definite based on the revised McDonald's criteria, ${ }^{19}$ the international consensus diagnostic criteria for $\mathrm{NMOSD}^{20}$ and the revised American college of rheumatology criteria, ${ }^{21}$ respectively. All patients were selected from the El-Kasr Aleini Hospital, Cairo, Egypt, where MS and NMOSD patients were recruited in the Neurology Department, MS unit, while NPSLE patients were recruited from the Rheumatology Department. Twenty-five healthy age- and sex-matched volunteers from the staff of the National Research Center (NRC) were also included as controls.

\section{Clinical Investigation}

All patients underwent the standard workflow; medical history, physical and neurological examination and brain, cervical and dorsal magnetic resonance imaging with contrast.

\section{Characterization of miRNA Expression Levels}

Blood samples (about $2 \mathrm{~mL}$ ) were drawn in the morning to avoid possible diurnal variation in miRNA levels. Serum was isolated within 2 hours from sample collection and then stored at $-20^{\circ} \mathrm{c}$ till further proceeding. miRNAs were isolated from the serum using miRNeasy kit (Qiagen, Germany) in accordance with the manufacturer's instructions. Synthetic C. elegans microRNA (cel-miR-39) (Qiagen, Germany) was added to normalize the expression levels of target miRNAs. Quantitative reverse transcription
(qRT) polymerase chain reaction (PCR) assays of miR-23a, miR-155 and miR-572 were performed using TaqMan ${ }^{\circledR}$ MicroRNA assay and kit (Applied Biosystems, USA) in accordance with the manufacturer's recommended protocols. The relative expression was determined using the $\Delta \Delta \mathrm{Ct}$ method, where fold change was calculated as

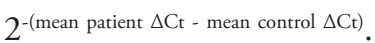

\section{Bioinformatics Analysis}

Ingenuity Pathway Analysis (IPA) software (Ingenuity Systems, California, USA) was utilized to retrieve genes involved in NMOSD pathogenesis as well as miR-23a validated targets involved in cellular immune response, then to construct and visualize possible interactions among extracted molecules.

\section{Statistical Analysis}

Statistical analyses were carried out using the Statistical Package for the Social Sciences (SPSS, version 18.0; IBM Corp., Chicago, USA, 2009). The Shapiro-Wilk test was used for normality testing. Categorical variables were expressed as the number of cases (\%) and compared using Pearson's chi-square $\left(\chi^{2}\right)$ test and Fisher's exact test, as applicable. Continuous normally distributed variables were represented as mean \pm standard deviation (SD) and compared using the independent Student's t-test or oneway analysis of variance (ANOVA) with post-hoc test. On the other hand, continuous variables showing nonnormal distribution were reported as median (interquartile range, IQR: 25th quartile to 75 th quartile) and pairwise comparisons were made using the non-parametric MannWhitney $U$ test. Absolute expression levels were used to compare patients with healthy subjects, while relative levels were employed when comparing patient groups. The correlations between miRNA expression levels were estimated using Spearman's rank correlation coefficient. Receiver operating characteristic (ROC) curve analysis was assessed to investigate the diagnostic/differential potential of the target miRNAs. The best cut-off value was determined as the point with the highest sum of specificity and sensitivity ${ }^{22}$ and Youden index was calculated to indicate the overall diagnostic benefit of each biomarker.

\section{Results}

Demographic and Clinical Data of the Cohort Study The study cohort involved 85 subjects, including $37 \mathrm{MS}$ patients, 13 NMOSD patients and 10 NPSLE patients, in addition to 25 healthy subjects. Among MS patients, 18 had RRMS (48.6\%), while 19 had SPMS (51.4\%). The majority of the enrolled subjects were females (68, $80 \%)$. No significant difference was documented between the study groups in terms of age $(P=0.131)$ or sex $(P=$ $0.563)$. The demographic and clinical features of the study groups are summarized in Table 1. 
Table 1. Demographic and Clinical Characteristics of the Cohort Study

\begin{tabular}{|c|c|c|c|c|c|c|}
\hline \multicolumn{7}{|c|}{ (A) Study Groups } \\
\hline \multicolumn{2}{|l|}{ Characteristic } & Healthy Subjects $(n=25)$ & \multirow{2}{*}{$\begin{array}{c}\text { MS Patients }(\mathbf{n}=37) \\
9(24.3 \%)\end{array}$} & $\begin{array}{l}\text { NMOSD Patients } \\
(n=13)\end{array}$ & $\begin{array}{l}\text { NPSLE Patients } \\
(\mathbf{n = 1 0})\end{array}$ & $P$ Value \\
\hline \multirow[t]{2}{*}{ Gender } & Male, n (\%) & $6(24.0 \%)$ & & $1(7.7 \%)$ & $1(10.0 \%)$ & \multirow{2}{*}{0.563} \\
\hline & Female, n (\%) & $19(76.0 \%)$ & $28(75.7 \%)$ & $12(92.3 \%)$ & $9(90.0 \%)$ & \\
\hline Age (y) & & $35.8 \pm 9.5$ & $33.1 \pm 8.8$ & $34.5 \pm 6.1$ & $28.3 \pm 7.2$ & 0.131 \\
\hline Age at onset (y) & & - & $25.3 \pm 7.0$ & $28.3 \pm 5.9$ & - & 0.166 \\
\hline Disease duration $(y)$ & & - & $7.8 \pm 6.1$ & $6.2 \pm 4.4$ & - & 0.401 \\
\hline \multicolumn{7}{|c|}{ (B) MS Subgroups } \\
\hline Characteristic & & \multicolumn{2}{|c|}{ RRMS ( $n=18)$} & \multicolumn{2}{|c|}{ SPMS $(n=19)$} & $P$ Value \\
\hline \multirow{2}{*}{ Gender } & Male, n (\%) & \multicolumn{2}{|c|}{$4(22.2 \%)$} & \multicolumn{2}{|c|}{$5(26.3 \%)$} & \\
\hline & Female, n (\%) & \multicolumn{2}{|c|}{$14(77.8 \%)$} & \multicolumn{2}{|c|}{$14(73.7 \%)$} & 1.000 \\
\hline Age $(y)$ & & \multicolumn{2}{|c|}{$28.2 \pm 6.8$} & \multicolumn{2}{|c|}{$37.7 \pm 8.0$} & $<0.001$ \\
\hline Age at onset (y) & & \multicolumn{2}{|c|}{$22.2 \pm 5.5$} & \multicolumn{2}{|c|}{$28.2 \pm 7.1$} & 0.008 \\
\hline Disease duration (y) & & \multicolumn{2}{|c|}{$5.9 \pm 4.4$} & \multicolumn{2}{|c|}{$9.5 \pm 6.9$} & 0.072 \\
\hline
\end{tabular}

MS, multiple sclerosis; NMOSD, neuromyelitis optica spectrum disorder; NPSLE, neuropsychiatric systemic lupus erythematosus; RRMS, relapsing-remitting multiple sclerosis; SPMS, secondary progressive multiple sclerosis.

Qualitative data are presented as the number of cases (\%), whereas quantitative data are represented as mean \pm SD.

Expression Patterns of miRNAs

The three miRNAs were dysregulated in MS patients compared to healthy subjects. However, miR-572 displayed significant downregulation $(P=0.027)$ when only the RRMS subgroup was considered. Conversely, in comparison to healthy subjects, target miRNAs were significantly downregulated in both NMOSD and NPSLE patients (Table 2, section A).

On the other hand, both miR-23a and miR-155 were found to be differentially expressed in MS vs. both NMOSD and NPSLE. miR-572 was significantly expressed only between SPMS and NMOSD patients $(P=0.048)$. None of the miRNAs showed differential expression between NMOSD and NPSLE, as well as RRMS and SPMS (Table 2, section B).

\section{Diagnostic Accuracy of miRNAs}

None of the analyzed miRNAs could singly distinguish MS patients from healthy subjects. However, miR$155+$ miR-572 and miR-23a + miR-155+miR-572 combinations indicated significant differentiation between MS and controls. When MS was stratified into subgroups, miR-572, alone or in combination with miR-23a, could distinguish RRMS from healthy subjects. On the contrary, the three miRNAs could discriminate both NMOSD and NPSLE patients from healthy subjects, whether in solitary, pair combined or triple combined use (Table 3, Figure 1).

Discriminatory Performance of miRNAs

Interestingly, miR-23a had a great potential to discriminate between MS and NMOSD with area under the curve (AUC) of 0.908 , a specificity of $100 \%$ and a sensitivity of $81.1 \%$. On the other hand, both miR-23a and miR-
155 distinguished patients with MS from those with NPSLE. miR-155 was the only single miRNA that could differentiate between NMOSD and NPSLE. miRNA combinations enhanced the differentiation potential in the study groups, particularly miR-155+miR-572 in case of NMOSD and NPSLE. Only miR-23a+miR-572 could discriminate between MS subgroups. The miRNA distinguishing ability showed no potential change when MS patients were stratified into subgroups (Table 4, Figure 2).

Correlation between miRNA Expression Levels

Significant correlations were detected between each two miRNAs in healthy subjects and MS patients. In both cases, the strongest correlation was reported between miR155 and miR-572. In case of patients with NMOSD and NPSLE, the only significant correlation was documented between miR-155 and miR-572 (Table 5).

\section{In Silico Analysis}

Our results revealed miR-23a as a candidate potential biomarker for discrimination between MS and NMOSD. Therefore, possible correlations between its potential targets and genes involved in NMOSD pathogenesis were generated using IPA to support our findings (Figure 3). Several direct correlations were demonstrated between the two sets. IL6R manifested as a common factor in the two groups.

\section{Discussion}

MS is a neuroinflammatory disease characterized by its relatively high prevalence in young adults. However, disease diagnosis, especially at early stages, still represents 
(A) Expression Levels in Terms of Absolute Quantitation $(\Delta \mathrm{ct})$

\begin{tabular}{|c|c|c|c|c|c|c|c|}
\hline miRNA & $\begin{array}{l}\text { Healthy Subjects } \\
(\mathbf{n}=25)\end{array}$ & $\begin{array}{l}\text { MS Patients } \\
(\mathbf{n}=37)\end{array}$ & $\begin{array}{l}\text { RRMS Patients } \\
(n=18)\end{array}$ & $\begin{array}{l}\text { SPMS Patients } \\
(\mathrm{n}=19)\end{array}$ & $\begin{array}{l}\text { NMOSD } \\
\text { Patients }(n=13)\end{array}$ & $\begin{array}{l}\text { NPSLE } \\
\text { Patients }(n=10)\end{array}$ & $P$ Value \\
\hline miR-23a & $3.36(1.54-4.26)$ & $3.14(1.91-4.93)$ & $2.85199(1.86-4.70)$ & $4.13(1.94-5.68)$ & $8.08(6.06-9.15)$ & $8.75(7.86-9.74)$ & $\begin{array}{l}0.672^{\mathrm{a}} \\
0.000^{\mathrm{b}} \\
0.000^{\mathrm{c}} \\
0.768^{\mathrm{d}} \\
0.325^{\mathrm{e}}\end{array}$ \\
\hline miR-155 & $3.14(1.06-5.57)$ & $5.06(.098-6.63)$ & $5.08(2.51-6.54)$ & $4.18(-1.77-7.02)$ & $7.49(5.28-8.26)$ & $8.03(7.47-9.51)$ & $\begin{array}{l}0.401^{\mathrm{a}} \\
0.001^{\mathrm{b}} \\
0.000^{\mathrm{c}} \\
0.192^{\mathrm{d}} \\
0.896^{\mathrm{e}}\end{array}$ \\
\hline miR-572 & $3.49(1.72-5.56)$ & $5.94(2.76-7.59)$ & $6.09(3.87-7.97)$ & $5.26(2.06-7.01)$ & $6.86(5.03-8.82)$ & $\begin{array}{l}6.37 \\
(5.30-8.78)\end{array}$ & $\begin{array}{l}0.059^{\mathrm{a}} \\
0.002^{\mathrm{b}} \\
0.005^{\mathrm{c}} \\
0.027^{\mathrm{d}} \\
0.325^{\mathrm{e}}\end{array}$ \\
\hline \multicolumn{8}{|c|}{ (B) Expression Levels in Terms of Relative Quantitation (RQ) } \\
\hline & $\begin{array}{l}\text { MS Patients } \\
(n=37)\end{array}$ & $\begin{array}{l}\text { NMOSD Patients } \\
(n=10)\end{array}$ & $\begin{array}{l}\text { NPSLE Patients } \\
(n=10)\end{array}$ & $P$ Value & $\begin{array}{l}\text { RRMS Patients } \\
(n=18)\end{array}$ & $\begin{array}{l}\text { SPMS Patients } \\
(n=19)\end{array}$ & $P$ Value \\
\hline miR-23a & $0.82(0.24-1.94)$ & $0.03(0.01-0.11)$ & $0.02(0.01-0.03)$ & $\begin{array}{l}0.000^{f^{*}} \\
0.000^{\mathrm{g}^{*}} \\
0.239^{\mathrm{h}}\end{array}$ & $1.01(0.28-2.01)$ & $0.41(0.14-1.90)$ & $\begin{array}{l}0.274^{\mathrm{i}} \\
0.000^{\mathrm{j}} \\
0.000^{\mathrm{k}} \\
0.000^{\mathrm{l}} \\
0.000^{\mathrm{m}}\end{array}$ \\
\hline miR-155 & $0.28(0.1-10.12)$ & $0.05(0.03-0.30)$ & $0.04(0.01-0.05)$ & $\begin{array}{l}0.012^{f^{*}} \\
0.000^{g^{*}} \\
0.082^{\mathrm{h}}\end{array}$ & $0.28(0.10-1.87)$ & $0.52(0.07-32.40)$ & $\begin{array}{l}0.429^{i} \\
0.028^{j} \\
0.002^{k} \\
0.025^{1} \\
0.002^{m}\end{array}$ \\
\hline miR-572 & $0.18(0.06-1.66)$ & $0.10(0.02-0.34)$ & $0.14(0.03-0.29)$ & $\begin{array}{l}0.113^{f} \\
0.242^{g} \\
0.880^{h}\end{array}$ & $0.16(0.04-0.82)$ & $0.29(0.09-2.67)$ & $\begin{array}{l}0.224^{i} \\
0.388^{j} \\
0.632^{k} \\
0.048^{\mathrm{l}} \\
0.108^{\mathrm{m}}\end{array}$ \\
\hline
\end{tabular}

MS, multiple sclerosis; NMOSD, neuromyelitis optica spectrum disorder; NPSLE, neuropsychiatric systemic lupus erythematosus; RRMS, relapsing-remitting multiple sclerosis; SPMS, secondary progressive multiple sclerosis.

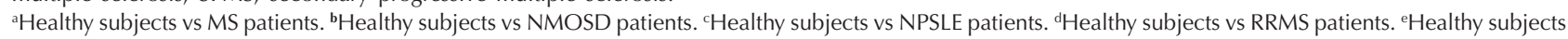
vs SPMS patients. ${ }^{\mathrm{M}} \mathrm{MS}$ patients vs NMOSD patients. ${ }^{\mathrm{g}} \mathrm{MS}$ patients vs NPSLE patients. ' $\mathrm{hMOSD}$ patients vs NPSLE patients. ${ }^{\mathrm{R} R} \mathrm{RMS}$ patients vs SPMS patients. ${ }^{\mathrm{i} R} \mathrm{RMS}$ patients vs NMOSD patients. ${ }^{k}$ RRMS patients vs NPSLE patients. 'SPMS patients vs NMOSD patients. ${ }^{\mathrm{m} S P M S}$ patients vs NPSLE patients.

Data are presented as median (interquartile range, IQR: 25th quartile to 75 th quartile).
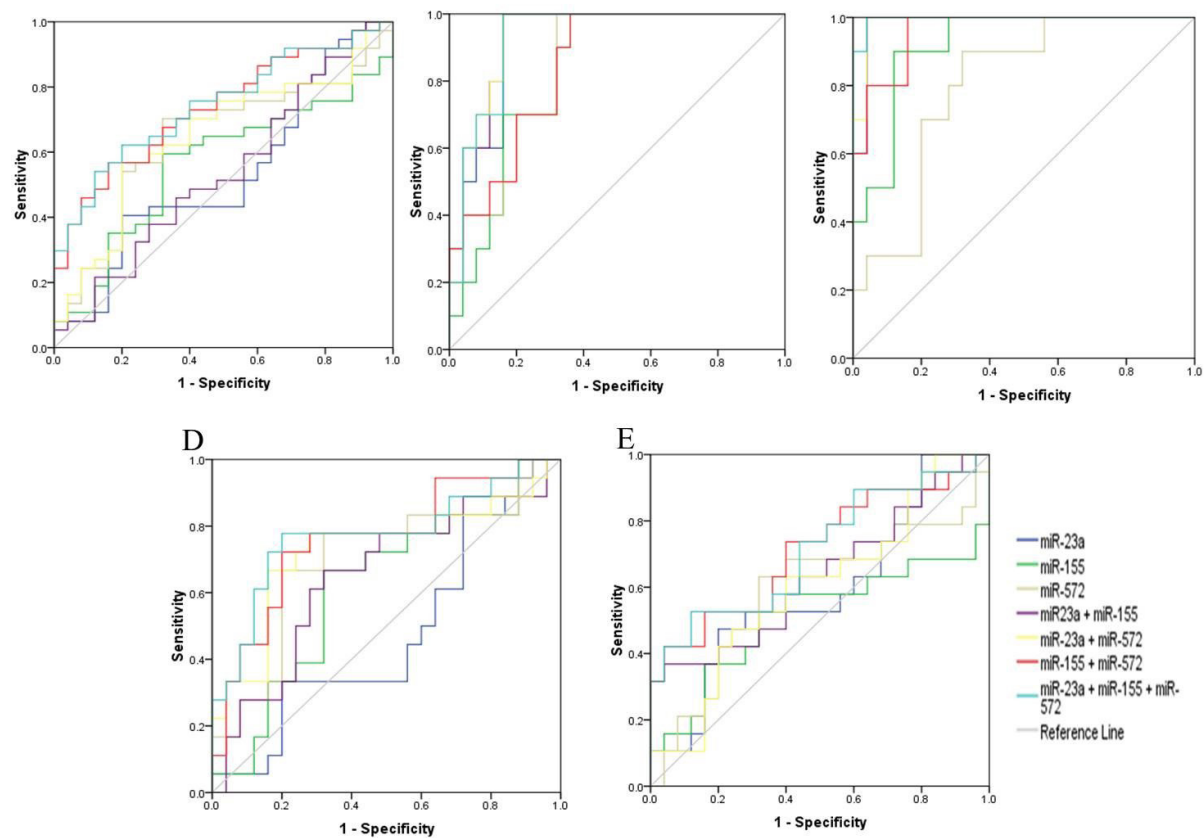

Figure 1. Receiver operating characteristic (ROC) curves of miRNA expression levels for diagnosing A) MS, B) NMOSD, C) NPSLE, D) RRMS and E) SPMS. 
Table 3. Performance of miRNA Expression Levels in Diagnosing Patient Groups

\begin{tabular}{|c|c|c|c|c|c|c|}
\hline Biomarker & AUC $(95 \% \mathrm{Cl})$ & $P$ Value & Best Cut-off Value & Sensitivity & Specificity & Youden's Index \\
\hline \multicolumn{7}{|c|}{ Healthy Subjects vs MS Patients } \\
\hline miR-23a & $0.532(0.382-0.681)$ & 0.672 & $\geq 4.3$ & $40.5 \%$ & $80.0 \%$ & $20.5 \%$ \\
\hline miR-155 & $0.563(0.418-0.709)$ & 0.401 & $\geq 4.1$ & $59.5 \%$ & $68.0 \%$ & $27.5 \%$ \\
\hline miR-572 & $0.642(0.501-0.784)$ & 0.059 & $\geq 4.2$ & $70.3 \%$ & $68.0 \%$ & $38.3 \%$ \\
\hline miR-23a + miR-155 & $0.537(0.389-0.685)$ & 0.621 & - & $45.9 \%$ & $64.0 \%$ & $9.5 \%$ \\
\hline miR-23a + miR-572 & $0.650(0.510-0.790)$ & 0.047 & - & $56.8 \%$ & $80 \%$ & $36.8 \%$ \\
\hline miR-155 + miR-572 & $0.735(0.612-0.858)$ & 0.002 & - & $56.8 \%$ & $84 \%$ & $40.8 \%$ \\
\hline miR-23a + miR-155 + miR-572 & $0.744(0.623-0.865)$ & 0.001 & - & $62.2 \%$ & $80 \%$ & $42.2 \%$ \\
\hline \multicolumn{7}{|c|}{ Healthy Subjects vs NMOSD Patients } \\
\hline miR-23a & $0.920(0.817-1.000)$ & $<0.001$ & $\geq 5.1$ & $100.0 \%$ & $84.0 \%$ & $84.0 \%$ \\
\hline miR-155 & $0.828(0.694-0.962)$ & 0.003 & $\geq 3.9$ & $100.0 \%$ & $64.0 \%$ & $64.0 \%$ \\
\hline miR-572 & $0.844(0.715-0.973)$ & 0.002 & $\geq 4.3$ & $100.0 \%$ & $68.0 \%$ & $68.0 \%$ \\
\hline miR-23a + miR-155 & $0.928(0.844-1.012)$ & $<0.001$ & - & $100.0 \%$ & $84.0 \%$ & $84.0 \%$ \\
\hline miR-23a + miR-572 & $0.932(0.850-1.014)$ & $<0.001$ & - & $100.0 \%$ & $84.0 \%$ & $84.0 \%$ \\
\hline miR-155 + miR-572 & $0.844(0.715-0.973)$ & 0.002 & - & $100.0 \%$ & $64.0 \%$ & $64.0 \%$ \\
\hline$m i R-23 a+m i R-155+m i R-572$ & $0.928(0.844-1.012)$ & $<0.001$ & - & $100.0 \%$ & $64.0 \%$ & $64.0 \%$ \\
\hline \multicolumn{7}{|c|}{ Healthy Subjects vs NPSLE Patients } \\
\hline miR-23a & $0.984(0.000-1.000)$ & $<0.001$ & $\geq 7.3$ & $100.0 \%$ & $96.0 \%$ & $96.0 \%$ \\
\hline miR-155 & $0.920(0.816-1.000)$ & $<0.001$ & $\geq 7.3$ & $90.0 \%$ & $88.0 \%$ & $78.0 \%$ \\
\hline miR-572 & $0.800(0.651-0.949)$ & $<0.001$ & $\geq 4.5$ & $90.0 \%$ & $68.0 \%$ & $58.0 \%$ \\
\hline miR-23a + miR-155 & $0.984(0.949-1.019)$ & $<0.001$ & - & $100.0 \%$ & $96.0 \%$ & $96.0 \%$ \\
\hline miR-23a + miR-572 & $0.988(0.960-1.016)$ & $<0.001$ & - & $100.0 \%$ & $96.0 \%$ & $96.0 \%$ \\
\hline miR-155 + miR-572 & $0.960(0.902-1.018)$ & $<0.001$ & - & $100.0 \%$ & $84.0 \%$ & $84.0 \%$ \\
\hline$m i R-23 a+m i R-155+m i R-572$ & $0.996(0.983-1.009)$ & $<0.001$ & - & $100.0 \%$ & $96.0 \%$ & $96.0 \%$ \\
\hline \multicolumn{7}{|c|}{ Healthy Subjects vs RRMS Patients } \\
\hline miR-23a & $0.473(0.295-0.652)$ & 0.768 & $\geq 4.4$ & $33.3 \%$ & $80 \%$ & $13.3 \%$ \\
\hline miR-155 & $0.618(0.444-0.791)$ & 0.192 & $\geq 4.1$ & $66.7 \%$ & $68 \%$ & $34.7 \%$ \\
\hline miR-572 & $0.700(0.532-0.868)$ & 0.027 & $\geq 5.7$ & $66.7 \%$ & $80 \%$ & $46.7 \%$ \\
\hline miR-23a + miR-155 & $0.649(0.477-0.821)$ & 0.099 & - & $66.7 \%$ & $68 \%$ & $34.7 \%$ \\
\hline miR-23a + miR-572 & $0.729(0.562-0.896)$ & 0.011 & - & $66.7 \%$ & $84 \%$ & $50.7 \%$ \\
\hline miR-155 + miR-572 & $0.760(0.609-0.911)$ & 0.004 & - & $72.2 \%$ & $80 \%$ & $52.2 \%$ \\
\hline miR-23a + miR-155 + miR-572 & $0.773(0.620-0.927)$ & 0.002 & - & $77.8 \%$ & $80 \%$ & $57.8 \%$ \\
\hline \multicolumn{7}{|c|}{ Healthy Subjects vs SPMS Patients } \\
\hline miR-23a & $0.587(0.413-0.761)$ & 0.325 & $\geq 4.3$ & $47.4 \%$ & $80 \%$ & $27.4 \%$ \\
\hline miR-155 & $0.512(0.322-0.701)$ & 0.896 & $\geq 5.8$ & $36.8 \%$ & $84 \%$ & $20.8 \%$ \\
\hline miR-572 & $0.587(0.407-0.767)$ & 0.325 & $\geq 4.3$ & $63.2 \%$ & $68 \%$ & $31.2 \%$ \\
\hline miR-23a + miR-155 & $0.636(0.464-0.807)$ & 0.126 & - & $36.8 \%$ & $96 \%$ & $32.8 \%$ \\
\hline miR-23a + miR-572 & $0.600(0.429-0.771)$ & 0.260 & - & $47.4 \%$ & $76 \%$ & $23.4 \%$ \\
\hline miR-155 + miR-572 & $0.712(0.552-0.871)$ & 0.017 & - & $42.1 \%$ & $96 \%$ & $38.1 \%$ \\
\hline miR-23a + miR-155 + miR-572 & $0.712(0.553-0.871)$ & 0.017 & - & $52.6 \%$ & $88 \%$ & $40.6 \%$ \\
\hline
\end{tabular}

MS, multiple sclerosis; NMOSD, neuromyelitis optica spectrum disorder; NPSLE, neuropsychiatric systemic lupus erythematosus; RRMS, relapsing-remitting multiple sclerosis; SPMS, secondary progressive multiple sclerosis.

a challenging process ${ }^{23}$ owing to several reasons; (1) early symptoms of MS are ill-defined and vague; (2) there are several symptomatic presentations of MS depending on which part of the CNS is affected; (3) the signs and symptoms of MS usually share considerable similarity with many other disease conditions; (4) there is no test or test sets that can confirm MS diagnosis; and 5-based on the most commonly used diagnostic criteria of MS (the revised McDonald Criteria), ${ }^{19}$ diagnosis of "definite" MS cannot be made after exclusion of all other possible diagnoses and requires that two different areas of the CNS should be affected on at least two separate occasions at least one month apart. As disease diagnosis is the patient's gateway to access therapeutic interventions, there is an urgent demand to investigate efficient diagnostic biomarkers during the early disease course.

miRNAs play pivotal roles in regulating the immune system biology, ${ }^{24}$ and it has been shown that changes in miRNA homeostasis mediate the pathophysiology of various inflammatory and autoimmune diseases and 
Table 4. Performance of miRNA Expression Levels in Distinguishing between Patient Groups

\begin{tabular}{|c|c|c|c|c|c|c|}
\hline Biomarker & AUC $(95 \% \mathrm{CI})$ & $P$ Value & Best Cut-off Value & Sensitivity & Specificity & Youden's Index \\
\hline \multicolumn{7}{|c|}{ MS vs NMOSD } \\
\hline miR-23a & $0.908(0.825-0.991)$ & $<0.001$ & $\geq 0.2$ & $81.1 \%$ & $100.0 \%$ & $81.1 \%$ \\
\hline miR-155 & $0.700(0.537-0.863)$ & 0.054 & $\geq 0.7$ & $40.5 \%$ & $100.0 \%$ & $40.5 \%$ \\
\hline miR-572 & $0.665(0.489-0.840)$ & 0.113 & $\geq 0.6$ & $32.4 \%$ & $100.0 \%$ & $32.4 \%$ \\
\hline miR-23a + miR-155 & $0.930(0.858-1.001)$ & $<0.001$ & - & $100.0 \%$ & $83.8 \%$ & $83.8 \%$ \\
\hline$m i R-23 a+m i R-572$ & $0.914(0.833-0.994)$ & $<0.001$ & - & $100.0 \%$ & $83.8 \%$ & $83.8 \%$ \\
\hline miR-23a + miR-155 + miR-572 & $0.930(0.858-1.002)$ & $<0.001$ & - & $100.0 \%$ & $83.8 \%$ & $83.8 \%$ \\
\hline \multicolumn{7}{|c|}{ NMOSD vs NPSLE } \\
\hline miR-23a & $0.700(0.447-0.953)$ & 0.131 & $\geq 0.1$ & $50.0 \%$ & $100.0 \%$ & $50.0 \%$ \\
\hline miR-155 & $0.760(0.547-0.973)$ & 0.049 & $\geq 0.1$ & $60.0 \%$ & $90.0 \%$ & $50.0 \%$ \\
\hline miR-572 & $0.480(0.215-0.745)$ & 0.880 & $\geq 0.1$ & $70.0 \%$ & $50.0 \%$ & $20.0 \%$ \\
\hline$m i R-23 a+m i R-155$ & $0.820(0.615-1.025)$ & 0.016 & - & $100.0 \%$ & $60.0 \%$ & $60.0 \%$ \\
\hline miR-23a + miR-572 & $0.760(0.546-0.974)$ & 0.049 & - & $100.0 \%$ & $50.0 \%$ & $50.0 \%$ \\
\hline miR-155 + miR-572 & $0.990(0.958-1.022)$ & $<0.001$ & - & $100.0 \%$ & $90.0 \%$ & $90.0 \%$ \\
\hline miR-23a + miR-155 + miR-572 & $0.990(0.958-1.022)$ & $<0.001$ & - & $100.0 \%$ & $90.0 \%$ & $90.0 \%$ \\
\hline \multicolumn{7}{|c|}{ RRMS vs NMOSD } \\
\hline miR-23a & $0.950(0.862-1.038)$ & $<0.001$ & $\geq 0.2$ & $88.9 \%$ & $100.0 \%$ & $88.9 \%$ \\
\hline miR-155 & $0.689(0.485-0.892)$ & 0.103 & $\geq 0.1$ & $83.3 \%$ & $50.0 \%$ & $33.3 \%$ \\
\hline miR-572 & $0.600(0.384-0.816)$ & 0.388 & $\geq 0.6$ & $27.8 \%$ & $100.0 \%$ & $27.8 \%$ \\
\hline miR-23a + miR-155 & $0.956(0.869-1.042)$ & $<0.001$ & - & $100.0 \%$ & $94.4 \%$ & $94.4 \%$ \\
\hline miR-23a + miR-572 & $0.956(0.869-1.042)$ & $<0.001$ & - & $100.0 \%$ & $94.4 \%$ & $94.4 \%$ \\
\hline miR-155 + miR-572 & $0.756(0.563-0.948)$ & 0.027 & - & $60.0 \%$ & $88.9 \%$ & $48.9 \%$ \\
\hline miR-23a + miR-155 + miR-572 & $0.967(0.899-1.034)$ & $<0.001$ & - & $100.0 \%$ & $94.4 \%$ & $94.4 \%$ \\
\hline \multicolumn{7}{|c|}{ SPMS vs NMOSD } \\
\hline miR-23a & $0.868(0.738-0.999)$ & $<0.001$ & $\geq 0.2$ & $73.7 \%$ & $100.0 \%$ & $73.7 \%$ \\
\hline miR-155 & $0.711(0.525-0.897)$ & 0.066 & $\geq 0.7$ & $47.4 \%$ & $100.0 \%$ & $47.4 \%$ \\
\hline miR-572 & $0.726(0.537-0.916)$ & 0.048 & $\geq 0.7$ & $36.8 \%$ & $100.0 \%$ & $36.8 \%$ \\
\hline miR-23a + miR-155 & $0.911(0.805-1.016)$ & $<0.001$ & - & $100.0 \%$ & $78.9 \%$ & $78.9 \%$ \\
\hline$m i R-23 a+m i R-572$ & $0.874(0.748-0.999)$ & 0.001 & - & $100.0 \%$ & $73.7 \%$ & $73.7 \%$ \\
\hline miR-155 + miR-572 & $0.747(0.566-0.929)$ & 0.031 & - & $90.0 \%$ & $52.6 \%$ & $42.6 \%$ \\
\hline \multicolumn{7}{|c|}{ MS vs NPSLE } \\
\hline miR-23a & $0.959(0.000-1.000)$ & $<0.001$ & $\geq 0.1$ & $91.9 \%$ & $100.0 \%$ & $91.9 \%$ \\
\hline miR-155 & $0.862(0.756-0.969)$ & $<0.001$ & $\geq 0.1$ & $81.1 \%$ & $90.0 \%$ & $71.1 \%$ \\
\hline miR-572 & $0.622(0.443-0.801)$ & 0.242 & $\geq 0.4$ & $37.8 \%$ & $90.0 \%$ & $27.8 \%$ \\
\hline miR-23a + miR-155 & $0.970(0.923-1.017)$ & $<0.001$ & - & $100.0 \%$ & $94.6 \%$ & $94.6 \%$ \\
\hline miR-23a + miR-572 & $0.968(0.923-1.013)$ & $<0.001$ & - & $100.0 \%$ & $91.9 \%$ & $91.9 \%$ \\
\hline miR-155 + miR-572 & $0.949(0.889-1.008)$ & $<0.001$ & - & $100.0 \%$ & $89.2 \%$ & $89.2 \%$ \\
\hline \multicolumn{7}{|c|}{ RRMS vs SPMS } \\
\hline miR23a & $0.605(0.421-0.790)$ & 0.274 & $\geq 2.3$ & $21.1 \%$ & $83.3 \%$ & $4.4 \%$ \\
\hline $\operatorname{miR} 155$ & $0.576(0.386-0.766)$ & 0.429 & $\geq 19.3$ & $31.6 \%$ & $100 \%$ & $31.6 \%$ \\
\hline $\operatorname{miR} 572$ & $0.617(0.435-0.799)$ & 0.224 & $\geq 0.28$ & $52.6 \%$ & $72.2 \%$ & $24.8 \%$ \\
\hline miR-23a + miR-155 & $0.678(0.503-0.854)$ & 0.064 & - & $63.2 \%$ & $77.8 \%$ & $41 \%$ \\
\hline miR-23a + miR-572 & $0.690(0.515-0.865)$ & 0.048 & - & $68.4 \%$ & $77.8 \%$ & $46.2 \%$ \\
\hline miR-155 + miR-572 & $0.617(0.434-0.800)$ & 0.224 & - & $42.1 \%$ & $83.3 \%$ & $25.4 \%$ \\
\hline miR-23a + miR-155 + miR-572 & $0.681(0.507-0.856)$ & 0.060 & - & $63.2 \%$ & $77.8 \%$ & $41 \%$ \\
\hline \multicolumn{7}{|c|}{ RRMS vs NPSLE } \\
\hline miR-23a & $0.972(0.914-1.030)$ & $<0.001$ & $\geq 0.1$ & $94.4 \%$ & $100.0 \%$ & $94.4 \%$ \\
\hline miR-155 & $0.867(0.728-1.005)$ & 0.002 & $\geq 0.1$ & $77.8 \%$ & $90.0 \%$ & $67.8 \%$ \\
\hline miR-572 & $0.556(0.337-0.774)$ & 0.632 & $\geq 0.5$ & $27.8 \%$ & $90.0 \%$ & $17.8 \%$ \\
\hline miR-23a + miR-155 & $0.972(0.914-1.030)$ & $<0.001$ & - & $100.0 \%$ & $94.4 \%$ & $94.4 \%$ \\
\hline miR-23a + miR-572 & $0.978(0.929-1.026)$ & $<0.001$ & - & $100.0 \%$ & $94.4 \%$ & $94.4 \%$ \\
\hline miR-155 + miR-572 & $0.989(0.960-1.018)$ & $<0.001$ & - & $90.0 \%$ & $100.0 \%$ & $90.0 \%$ \\
\hline$m i R-23 a+m i R-155+m i R-572$ & $0.994(0.977-1.012)$ & $<0.001$ & - & $100.0 \%$ & $94.4 \%$ & $94.4 \%$ \\
\hline \multicolumn{7}{|c|}{ SPMS vs NPSLE } \\
\hline miR-23a & $0.947(0.868-1.027)$ & $<0.001$ & $\geq 0.1$ & $89.5 \%$ & $100.0 \%$ & $89.5 \%$ \\
\hline miR-155 & $0.858(0.723-0.992)$ & 0.002 & $\geq 0.1$ & $78.9 \%$ & $90.0 \%$ & $68.9 \%$ \\
\hline miR-572 & $0.684(0.487-0.882)$ & 0.108 & $\geq 0.3$ & $52.6 \%$ & $80.0 \%$ & $32.6 \%$ \\
\hline miR-23a + miR-155 & $0.995(0.978-1.012)$ & $<0.001$ & - & $100.0 \%$ & $94.7 \%$ & $94.7 \%$ \\
\hline$m i R-23 a+m i R-572$ & $0.958(0.891-1.025)$ & $<0.001$ & - & $100.0 \%$ & $89.5 \%$ & $89.5 \%$ \\
\hline miR-155 + miR-572 & $0.932(0.841-1.023)$ & $<0.001$ & - & $100.0 \%$ & $84.2 \%$ & $84.2 \%$ \\
\hline
\end{tabular}

MS, multiple sclerosis; NMOSD, neuromyelitis optica spectrum disorder; NPSLE, neuropsychiatric systemic lupus erythematosus; RRMS, relapsing-remitting multiple sclerosis; SPMS, secondary progressive multiple sclerosis. 


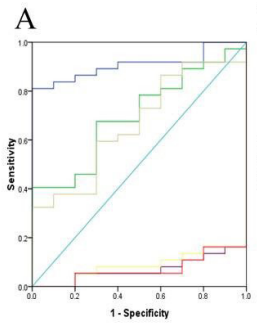

B
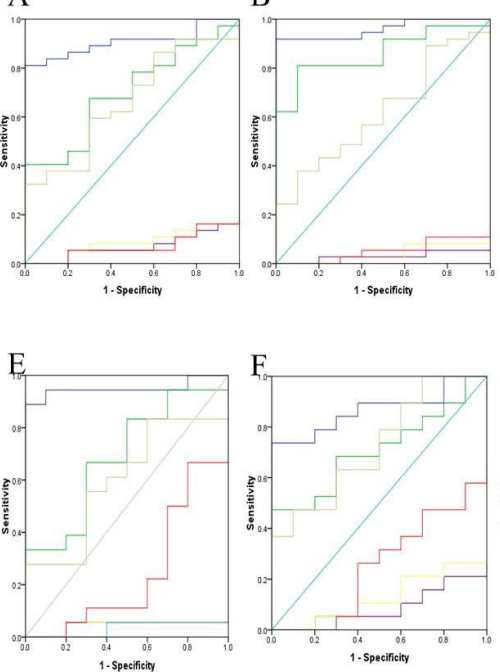

$\mathrm{C}$

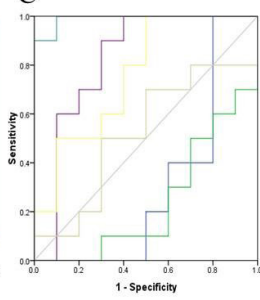

G

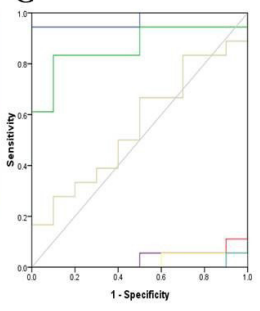

$\mathrm{D}$

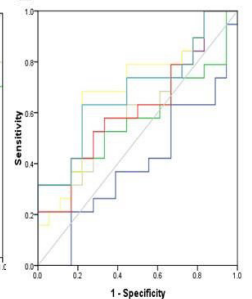

$\mathrm{H}$

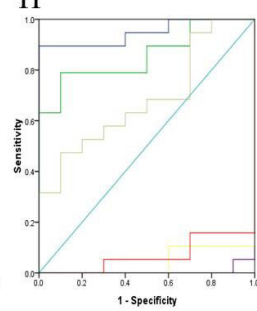

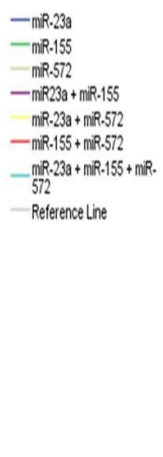

Figure 2. Receiver Operating Characteristic (ROC) Curves of miRNA Expression Levels Fold Change for Differentiating MS from NMOSD (A), MS from NPSLE (B), NMOSD from NPSLE (C), RRMS from SPMS (D), RRMS from NMOSD (E), SPMS from NMOSD (F), RRMS from NPSLE (G) and SPMS from NPSLE (H).

strongly correlate with disease phenotype and severity. ${ }^{25} \mathrm{On}$ the other hand, miRNA dysregulation has been reported in several neurologic disorders and circulating miRNAs have been proposed as useful indicators for diagnosis and prognosis of different neurological diseases. ${ }^{26}$ Regarding MS, It has been shown that at least 62 miRNAs were dysregulated in plasma/serum of disease patients. ${ }^{27}$ So far, no specific miRNAs have been utilized as biomarkers for MS, ${ }^{28}$ even though miRNA signature profiles have already been employed for the diagnosis of rheumatoid arthritis ${ }^{29}$ and inflammatory bowel disease. ${ }^{30}$ In the last few years, special interest is being dedicated to investigating altered miRNA levels in MS patients that may serve as potential biomarkers for diagnosing the disease and differentiating its subtypes, quantifying disease severity, monitoring its activity and evaluating treatment responses. ${ }^{27,31,32}$

In this preliminary study, the expression levels of three miRNAs (miR-23a, miR-155, and miR-572) were evaluated in the sera of Egyptian patients experiencing MS. They revealed no significant dysregulation in total MS patients. However, miR-572 was significantly expressed in SPMS compared to healthy subjects. Moreover, certain miRNA combinations demonstrated a considerable potential for diagnosing MS and its subtypes. Consistently, a profiling miRNA study in the cerebrospinal fluid of MS patients showed that the composite use of miRNAs holds greater promise for diagnosing MS and also distinguishing RRMS from PPMS. ${ }^{33}$

Importantly, there is lack of replication between the independent miRNA studies in MS and in accordance to our findings, some profiling studies showed no differential expression for miR-23a, miR-155 and miR-572 in MS patients. ${ }^{34,35}$ This discrepancy of miRNA expression levels among different studies might be attributed to variations in the genetic background and clinical criteria of enrolled patients, treatment regimens, tissues analyzed, and experimental and statistical approaches applied. Importantly, application of two miRNA profiling methods for the same cohort obtained different results. ${ }^{36}$ On the other hand, potentially different expression of substantial miRNA proportion has been detected between Africans and Europeans, proposing that genetic variations might influence the miRNA profile. ${ }^{37}$

The transition point from RRMS to SPMS is hardly defined and often retrospectively determined. ${ }^{38}$ Research is underway concerning potential laboratory and imaging biomarkers, which can distinguish SPMS from RRMS and even predict the transition from RRMS to SPMS; however, no suggested biomarker has been validated yet. ${ }^{6}$ In this study, miR-23a in combination with miR-572 was differently expressed in relapsing and progressive disease phases.

The clinical manifestations of MS share considerable similarity with several other diseases. In particular, NMOSD, previously believed to be a form of MS, is highly similar and may be indistinct from MS. ${ }^{39}$ Systemic lupus erythematosus (SLE) may also present relapsing, multifocal neurologic manifestations comparable to those of MS, known as neuropsychiatric SLE (NPSLE)..$^{40}$ Currently, no laboratory biomarker exists for establishing an accurate diagnosis for any of these disease entities. However, classical MS therapies may not be effective or even worsen disease severity in such disorders. In this regard, accurate reliable biomarkers that could help in their discrimination are urgently needed.

In the current study, the expression levels of the investigated miRNAs were also analyzed in the sera of patients with NMOSD and NPSLE. We demonstrated 
Table 5. Correlation between miRNA Expression Levels in the Study Groups

\begin{tabular}{|c|c|c|c|c|c|}
\hline Groups & Variables & & miR23a & miR155 & miR572 \\
\hline \multirow{6}{*}{$\begin{array}{l}\text { Healthy } \\
\text { subjects }\end{array}$} & \multirow{2}{*}{ miR23a } & $\mathrm{R}$ & - & 0.409 & 0.491 \\
\hline & & $P$ value & - & 0.042 & 0.013 \\
\hline & \multirow{2}{*}{ miR155 } & $\mathrm{R}$ & 0.409 & - & 0.864 \\
\hline & & $P$ value & 0.042 & - & 0.000 \\
\hline & \multirow{2}{*}{ miR572 } & $\mathrm{R}$ & 0.491 & 0.864 & - \\
\hline & & $P$ value & 0.013 & 0.000 & - \\
\hline \multirow{6}{*}{ MS patients } & \multirow{2}{*}{ miR23a } & $\mathrm{R}$ & - & 0.458 & 0.519 \\
\hline & & $P$ value & - & 0.004 & 0.001 \\
\hline & \multirow{2}{*}{ miR155 } & $\mathrm{R}$ & 0.458 & - & 0.910 \\
\hline & & $P$ value & 0.004 & - & 0.000 \\
\hline & \multirow{2}{*}{ miR572 } & $\mathrm{R}$ & 0.519 & 0.910 & - \\
\hline & & $P$ value & 0.001 & 0.000 & - \\
\hline \multirow{6}{*}{$\begin{array}{l}\text { NMOSD } \\
\text { patients }\end{array}$} & \multirow{2}{*}{ miR23a } & $R$ & - & 0.247 & 0.164 \\
\hline & & $P$ value & - & 0.415 & 0.651 \\
\hline & \multirow{2}{*}{ miR155 } & $\mathrm{R}$ & 0.247 & - & 0.915 \\
\hline & & $P$ value & 0.415 & - & 0.000 \\
\hline & \multirow{2}{*}{ miR572 } & $\mathrm{R}$ & 0.164 & 0.915 & - \\
\hline & & $P$ value & 0.651 & 0.000 & - \\
\hline \multirow{6}{*}{$\begin{array}{l}\text { NPSLE } \\
\text { patients }\end{array}$} & \multirow{2}{*}{ miR23a } & $\mathrm{R}$ & - & -0.285 & -0.467 \\
\hline & & $P$ value & - & 0.425 & 0.174 \\
\hline & \multirow{2}{*}{ miR155 } & $\mathrm{R}$ & -0.285 & - & 0.782 \\
\hline & & $P$ value & 0.425 & - & 0.008 \\
\hline & \multirow{2}{*}{ miR572 } & $\mathrm{R}$ & -0.467 & 0.782 & - \\
\hline & & $P$ value & 0.174 & 0.008 & - \\
\hline
\end{tabular}

that the analyzed miRNAs exhibited significant downregulation in both NMOSD and NPSLE patients compared to control subjects. Furthermore, the expression levels of both miR-23a and miR-155 revealed significant differences in MS vs. NPSLE patients. We have also previously reported differential expression of 3 miRNAs between MS and NPSLE. ${ }^{41}$ On the other hand, miR23a showed a high diagnostic value for distinguishing NMOSD from MS. In a previous report by our group, none of the selected miRNAs showed significant variations between NMOSD and MS..$^{41}$ Moreover, other studies have also failed to detect any differential miRNA expressions in sera of patients with MS vs. NMOSD. ${ }^{42,43}$ This highlights the clinical importance of miR-23a which may act as an exceptional biomarker for distinguishing between these closely related disease entities.

In conclusion, our results suggest that circulating miRNAs might provide useful insights for the differential diagnosis of neuroinflammatory diseases. However, a potential limitation of our study is the restricted number subjects in each group. Therefore, further studies on larger and welldefined patient groups are obviously required to explore the validity of our preliminary findings and consequently, the clinical usefulness of the target miRNAs for distinguishing between the enrolled patients. Furthermore, there is

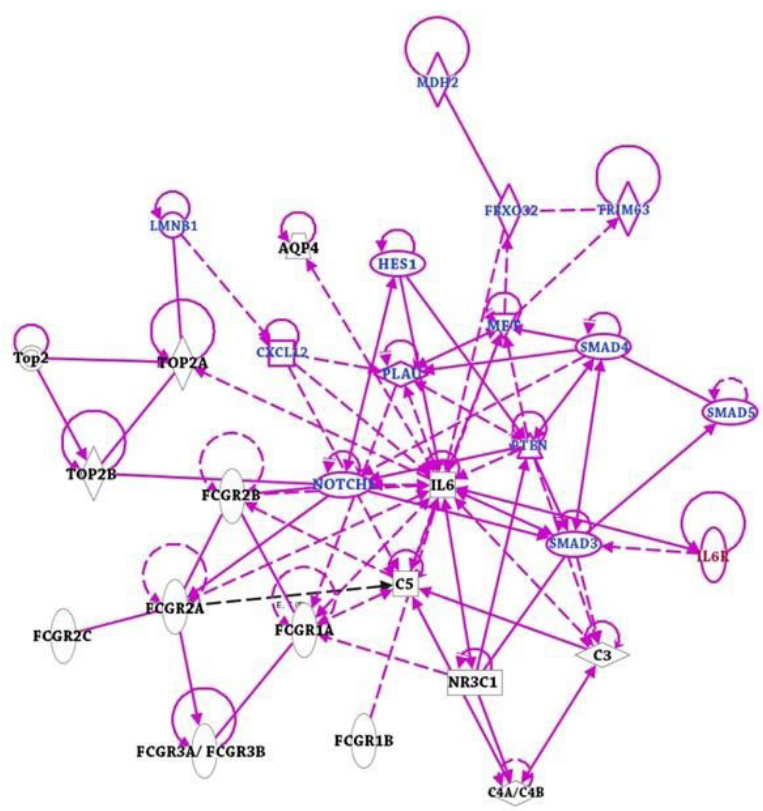

Figure 3. Interaction Network between Potential miR-23a Targets and Genes Involved in NMOSD Pathogenesis. The network was generated through IPA. Only direct interactions are shown. Every interaction is supported by at least one reference. Genes involved in NMOSD pathogenesis are shown in black; potential miR-23a targets in blue; and common molecules in red.

urgent need for method standardization regarding miRNA extraction, quantification, and expression estimation to enable comparing results from multiple laboratories and ultimately defining the optimal miRNA composite, not only for diagnosis of different disease phenotypes, but also for the discrimination of clinically overlapping disease structures.

\section{Authors' Contribution}

ME and WS conceived the original idea. NK and BS selected and investigated the enrolled cases. WS, NE and MR carried out the experiment under supervision of ME. HE and WS carried out the statistical analysis. WS wrote the manuscript with support from ME.

\section{Conflict of Interest Disclosures}

The authors declare that they have no conflict of interest.

\section{Ethical Statement}

Written informed consent was obtained from all participants and the study was approved by the Medical Research Ethics Committee (MREC) at the National Research Centre.

\section{Acknowledgements}

The work was supported by grant \#11010167 from the National Research Centre (NRC).

\section{References}

1. Compston A, Coles A. Multiple sclerosis. Lancet. 2008; 372(9648):1502-17. doi: 10.1016/S0140-6736(08)61620-7.

2. Visser EM, Wilde K, Wilson JF, Yong KK, Counsell CE. A new prevalence study of multiple sclerosis in Orkney, Shetland and Aberdeen city. J Neurol Neurosurg Psychiatry. 2012;83(7):71924. doi: 10.1136/jnnp-2011-301546.

3. Browne P, Chandraratna D, Angood C, Tremlett H, Baker C, Taylor BV, et al. Atlas of Multiple Sclerosis 2013: A growing 
global problem with widespread inequity. Neurology. 2014;83(11):1022-4. doi: 10.1212/wnl.0000000000000768.

4. Heydarpour P, Khoshkish S, Abtahi S, Moradi-Lakeh M, Sahraian MA. Multiple Sclerosis Epidemiology in Middle East and North Africa: A Systematic Review and MetaAnalysis. Neuroepidemiology. 2015;44(4):232-44. doi: 10.1159/000431042.

5. El-Tallawy HN, Farghaly WM, Badry R, Metwally NA, Shehata GA, Rageh TA, et al. Prevalence of multiple sclerosis in Al Quseir city, Red Sea Governorate, Egypt. Neuropsychiatr Dis Treat. 2016;12:155-8. doi: 10.2147/NDT.S87348.

6. Katz Sand I. Classification, diagnosis, and differential diagnosis of multiple sclerosis. Curr Opin Neurol. 2015;28(3):193-205. doi: 10.1097/WCO.0000000000000206.

7. Zakaria M, Zamzam DA, Abdel Hafeez MA, Swelam MS, Khater SS, Fahmy MF, et al. Clinical characteristics of patients with multiple sclerosis enrolled in a new registry in Egypt. Mult Scler Relat Disord. 2016;10:30-5. doi: 10.1016/j. msard.2016.06.013.

8. Fenoglio C, Ridolfi E, Cantoni C, De Riz M, Bonsi R, Serpente $M$, et al. Decreased circulating miRNA levels in patients with primary progressive multiple sclerosis. Mult Scler. 2013;19(14):1938-42. doi: 10.1177/1352458513485654.

9. Ridolfi E, Fenoglio C, Cantoni C, Calvi A, De Riz M, Pietroboni A, et al. Expression and Genetic Analysis of MicroRNAs Involved in Multiple Sclerosis. Int J Mol Sci. 2013;14(3):437584. doi: 10.3390/ijms14034375.

10. Jernas M, Malmestrom C, Axelsson M, Nookaew I, Wadenvik $\mathrm{H}$, Lycke J, et al. MicroRNA regulate immune pathways in T-cells in multiple sclerosis (MS). BMC Immunol. 2013;14:32. doi: 10.1186/1471-2172-14-32.

11. Junker A, Krumbholz M, Eisele S, Mohan $\mathrm{H}$, Augstein F, Bittner $R$, et al. MicroRNA profiling of multiple sclerosis lesions identifies modulators of the regulatory protein CD47. Brain. 2009;132(Pt 12):3342-52. doi: 10.1093/brain/awp300.

12. Zhang J, Cheng Y, Cui W, Li M, Li B, Guo L. MicroRNA-155 modulates Th1 and Th17 cell differentiation and is associated with multiple sclerosis and experimental autoimmune encephalomyelitis. J Neuroimmunol. 2014;266(1-2):56-63. doi: 10.1016/j.jneuroim.2013.09.019.

13. Waschbisch A, Atiya M, Linker RA, Potapov S, Schwab S, Derfuss T. Glatiramer acetate treatment normalizes deregulated microRNA expression in relapsing remitting multiple sclerosis. PLoS One. 2011;6(9):e24604. doi: 10.1371/journal. pone.0024604.

14. Moore CS, Rao VT, Durafourt BA, Bedell BJ, Ludwin SK, BarOr A, et al. miR-155 as a multiple sclerosis-relevant regulator of myeloid cell polarization. Ann Neurol. 2013;74(5):709-20. doi: 10.1002/ana.23967.

15. Lopez-Ramirez MA, Wu D, Pryce G, Simpson JE, Reijerkerk A, King-Robson J, et al. MicroRNA-155 negatively affects bloodbrain barrier function during neuroinflammation. FASEB J. 2014;28(6):2551-65. doi: 10.1096/fj.13-248880.

16. Noorbakhsh F, Ellestad KK, Maingat F, Warren KG, Han MH, Steinman L, et al. Impaired neurosteroid synthesis in multiple sclerosis. Brain. 2011;134(Pt 9):2703-21. doi: 10.1093/brain/ awr200.

17. Siegel SR, Mackenzie J, Chaplin G, Jablonski NG, Griffiths L. Circulating microRNAs involved in multiple sclerosis. Mol Biol Rep. 2012;39(5):6219-25. doi: 10.1007/s11033-0111441-7.

18. Mancuso R, Hernis A, Agostini S, Rovaris M, Caputo D, Clerici M. MicroRNA-572 expression in multiple sclerosis patients with different patterns of clinical progression. J Transl Med. 2015;13:148. doi: 10.1186/s12967-015-0504-2.

19. Polman $\mathrm{CH}$, Reingold SC, Banwell B, Clanet $\mathrm{M}$, Cohen JA, Filippi $M$, et al. Diagnostic criteria for multiple sclerosis: 2010 revisions to the McDonald criteria. Ann Neurol.
2011:69(2):292-302. doi: 10.1002/ana.22366

20. Wingerchuk DM, Banwell B, Bennett JL, Cabre P, Carroll W, Chitnis $\mathrm{T}$, et al. International consensus diagnostic criteria for neuromyelitis optica spectrum disorders. Neurology. 2015;85(2):177-89. doi: 10.1212/WNL.0000000000001729.

21. Hochberg MC. Updating the American College of Rheumatology revised criteria for the classification of systemic lupus erythematosus. Arthritis Rheum. 1997;40(9):1725. doi: $\quad$ 10.1002/1529-0131(199709)40:9\&It;1725::AIDART29\&gt;3.0.CO;2-Y.

22. Fluss $R$, Faraggi $D$, Reiser B. Estimation of the Youden Index and its associated cutoff point. Biom J. 2005;47(4):458-72.

23. Solomon AJ, Klein EP, Bourdette D. "Undiagnosing" multiple sclerosis: the challenge of misdiagnosis in MS. Neurology. 2012;78(24):1986-91. doi: 10.1212/ WNL.0b013e318259e1b2.

24. O'Connell RM, Rao DS, Chaudhuri AA, Baltimore D. Physiological and pathological roles for microRNAs in the immune system. Nat Rev Immunol. 2010;10(2):111-22. doi: 10.1038/nri2708.

25. Chen JQ, Papp G, Szodoray P, Zeher M. The role of microRNAs in the pathogenesis of autoimmune diseases. Autoimmun Rev. 2016;15(12):1171-80. doi:10.1016/j.autrev.2016.09.003.

26. Sheinerman KS, Umansky SR. Circulating cell-free microRNA as biomarkers for screening, diagnosis and monitoring of neurodegenerative diseases and other neurologic pathologies. Front Cell Neurosci. 2013;7:150. doi: 10.3389/ fncel.2013.00150.

27. Jagot F, Davoust N. Is It worth Considering Circulating microRNAs in Multiple Sclerosis?. Front Immunol. 2016;7:129. doi: 10.3389/fimmu.2016.00129.

28. Huang Q, Xiao B, Ma X, Qu M, Li Y, Nagarkatti P, et al. MicroRNAs associated with the pathogenesis of multiple sclerosis. J Neuroimmunol. 2016;295-296:148-61. doi: 10.1016/j.jneuroim.2016.04.014.

29. Ormseth MJ, Solus JF, Vickers KC, Oeser AM, Raggi P, Stein CM. Utility of Select Plasma MicroRNA for Disease and Cardiovascular Risk Assessment in Patients with Rheumatoid Arthritis. J Rheumatol. 2015;42(10):1746-51. doi: 10.3899/ jrheum.150232.

30. Hubenthal M, Hemmrich-Stanisak G, Degenhardt F, Szymczak S, Du Z, Elsharawy A, et al. Sparse Modeling Reveals miRNA Signatures for Diagnostics of Inflammatory Bowel Disease. PLoS One. 2015;10(10):e0140155. doi: 10.1371/journal. pone.0140155.

31. Ma X, Zhou J, Zhong Y, Jiang L, Mu P, Li Y, et al. Expression, regulation and function of microRNAs in multiple sclerosis. Int J Med Sci. 2014;11(8):810-810.7150/ijms.8647.

32. Gandhi R. miRNA in multiple sclerosis: search for novel biomarkers. Mult Scler. 2015;21(9):1095-103. doi: 10.1177/1352458515578771.

33. Haghikia A, Haghikia A, Hellwig K, Baraniskin A, Holzmann A, Decard BF, et al. Regulated microRNAs in the CSF of patients with multiple sclerosis: a case-control study. Neurology. 2012;79(22):2166-70. doi: 10.1212/ WNL.0b013e3182759621.

34. Luo D, Fu J. Identifying characteristic miRNAs-genes and risk pathways of multiple sclerosis based on bioinformatics analysis. Oncotarget. 2018;9(4):5287-300. doi: 10.18632/ oncotarget.23866.

35. Piket E, Zheleznyakova GY, Kular L, Jagodic M. Small non-coding RNAs as important players, biomarkers and therapeutic targets in multiple sclerosis: A comprehensive overview. J Autoimmun. 2019;101:17-25. doi: 10.1016/j. jaut.2019.04.002.

36. Keller A, Leidinger $\mathrm{P}$, Steinmeyer $\mathrm{F}$, Stahler C, Franke A, Hemmrich-Stanisak G, et al. Comprehensive analysis of microRNA profiles in multiple sclerosis including next- 
generation sequencing. Mult Scler. 2014;20(3):295-303. doi: 10.1177/1352458513496343.

37. Huang RS, Gamazon ER, Ziliak D, Wen Y, Im HK, Zhang W, et al. Population differences in microRNA expression and biological implications. RNA Biol. 2011;8(4):692-701. doi: 10.4161/rna.8.4.16029.

38. Katz Sand I, Krieger S, Farrell C, Miller AE. Diagnostic uncertainty during the transition to secondary progressive multiple sclerosis. Mult Scler. 2014;20(12):1654-7. doi: $10.1177 / 1352458514521517$.

39. Trebst C, Jarius S, Berthele A, Paul F, Schippling S, Wildemann $\mathrm{B}$, et al. Update on the diagnosis and treatment of neuromyelitis optica: recommendations of the Neuromyelitis Optica Study Group (NEMOS). J Neurol. 2014;261(1):1-16. doi: 10.1007/ s00415-013-7169-7.

40. Ferreira S, D'Cruz DP, Hughes GR. Multiple sclerosis, neuropsychiatric lupus and antiphospholipid syndrome: where do we stand? Rheumatology. 2005;44(4):434-42. doi: 10.1093/rheumatology/keh532.

41. Sharaf-Eldin WE, Kishk NA, Gad YZ, Hassan H, Ali MAM, Zaki MS, et al. Extracellular miR-145, miR-223 and miR326 expression signature allow for differential diagnosis of immune-mediated neuroinflammatory diseases. J Neurol Sci. 2017;383:188-98. doi: 10.1016/j.jns.2017.11.014.

42. Keller A, Leidinger P, Meese E, Haas J, Backes C, Rasche L, et al. Next-generation sequencing identifies altered whole blood microRNAs in neuromyelitis optica spectrum disorder which may permit discrimination from multiple sclerosis. J Neuroinflammation. 2015;12:196. doi: 10.1186/s12974-0150418-1.

43. Chen J, Zhu J, Wang Z, Yao X, Wu X, Liu F, et al. MicroRNAs Correlate with Multiple Sclerosis and Neuromyelitis Optica Spectrum Disorder in a Chinese Population. Med Sci Monit. 2017;23:2565-2583. doi: 10.12659/msm.904642. 